\title{
Decay of metastable phases in a model for the catalytic oxidation of $\mathrm{CO}$
}

\author{
Erik Machado and Gloria M. Buendía \\ Physics Department, Universidad Simón Bolívar, \\ Apartado 89000, Caracas 1080, Venezuela \\ Per Arne Rikvold \\ Center for Materials Research and Technology, School of Computational Science, \\ National High Magnetic Field Laboratory, and Department of Physics, \\ Florida State University, Tallahassee, FL 32306-4350, USA
}

(Dated: June 13, 2021) 


\begin{abstract}
We study by kinetic Monte Carlo simulations the dynamic behavior of a Ziff-Gulari-Barshad model with $\mathrm{CO}$ desorption for the reaction $\mathrm{CO}+\mathrm{O} \rightarrow \mathrm{CO}_{2}$ on a catalytic surface. Finite-size scaling analysis of the fluctuations and the fourth-order order-parameter cumulant show that below a critical $\mathrm{CO}$ desorption rate, the model exhibits a nonequilibrium first-order phase transition between low and high CO coverage phases. We calculate several points on the coexistence curve. We also measure the metastable lifetimes associated with the transition from the low CO coverage phase to the high $\mathrm{CO}$ coverage phase, and vice versa. Our results indicate that the transition process follows a mechanism very similar to the decay of metastable phases associated with equilibrium first-order phase transitions and can be described by the classic Kolmogorov-Johnson-Mehl-Avrami theory of phase transformation by nucleation and growth. In the present case, the desorption parameter plays the role of temperature, and the distance to the coexistence curve plays the role of an external field or supersaturation. We identify two distinct regimes, depending on whether the system is far from or close to the coexistence curve, in which the statistical properties and the system-size dependence of the lifetimes are different, corresponding to multidroplet or single-droplet decay, respectively. The crossover between the two regimes approaches the coexistence curve logarithmically with system size, analogous to the behavior of the crossover between multidroplet and single-droplet metastable decay near an equilibrium first-order phase transition.

PACS numbers: 82.65.+r, 64.60.Ht, 82.20.Wt, 05.40.-a
\end{abstract}




\section{INTRODUCTION}

The study of phase transitions and critical phenomena in nonequilibrium systems is a subject of great interest. In particular, the study of surface reaction models has attracted considerable attention [1]. These models not only exhibit rich and complex behavior, but they can also explain a wide range of experimental results associated with catalysis and could be very useful for designing more efficient processes. The potential applications of improved catalytic reactions are a powerful reason to pursue this line of research [2]. Unlike the decay of metastable phases near first-order equilibrium phase transitions, the decay of metastable phases near nonequilibrium phase transitions still lacks a well-established theoretical framework.

The Ziff, Gulari, and Barshad (ZGB) model is a lattice-gas adsorption-reaction model that describes some kinetic aspects of the catalytic oxidation of carbon monoxide on a crystal surface 3]. The ZGB model assumes that the reaction between $\mathrm{CO}$ and $\mathrm{O}_{2}$ on a surface proceeds according to the Langmuir-Hinshelwood process:

$$
\begin{aligned}
\mathrm{CO}(\mathrm{g})+\mathrm{S} & \rightarrow \mathrm{CO}(\mathrm{a}) \\
\mathrm{O}_{2}+2 \mathrm{~S} & \rightarrow 2 \mathrm{O}(\mathrm{a}) \\
\mathrm{CO}(\mathrm{a})+\mathrm{O}(\mathrm{a}) & \rightarrow \mathrm{CO}_{2}(\mathrm{~g})+2 \mathrm{~S}
\end{aligned}
$$

where $\mathrm{S}$ is an empty site on the surface, and $(\mathrm{g})$ and (a) refer to the gas and adsorbed phase, respectively. The process is controlled by a single parameter $y$, which represents the probability that the next molecule arriving at the surface is CO, i.e., it is proportional to the partial pressure of CO. The model exhibits two kinetic phase transitions, a continuous one at $y=y_{1}$ and a discontinuous one at $y=y_{2}$, where $y_{1}<y_{2}$. When $y<y_{1}$, all the sites become occupied by oxygen, the so-called oxygen-poisoned state. If $y>y_{2}$, all the sites become occupied by $\mathrm{CO}$ molecules, the so-called CO-poisoned state. Real systems do not possess an oxygen-poisoned state because oxygen does not impede the adsorption of CO. However, transitions between states of low and high CO coverage $\theta_{\mathrm{CO}}$ (where $\theta_{\mathrm{CO}}$

is the fraction of surface sites occupied by $\mathrm{CO}$ ) have been observed experimentally [4, [5]. At low temperatures, as $y$ increases, there is a discontinuous increase in $\theta_{\mathrm{CO}}$, accompanied by a discontinuous drop in the $\mathrm{CO}_{2}$ production rate. Above a critical temperature the discontinuities disappear, and the $\mathrm{CO}_{2}$ production decreases continuously. This type of 
behavior can be reproduced by modifying the ZGB model to include a CO desorption rate, $k$ [6, 7, 8, 9], a model we for brevity will call the ZGB-k model [10]. For this model, there is a distinction between high and low CO-coverage phases only for $k$ below a critical value $k_{c}$, while above $k_{c}$ the CO coverage varies smoothly with $y$. Thus, the transition value $y_{2}$ becomes a function of $k$, corresponding to a coexistence curve $y_{2}(k)$ that terminates at the critical point $y_{2}\left(k_{c}\right)$ [11, 12]. The ZGB-k model does not have a totally poisoned CO state, and hysteresis is observed in $\theta_{\mathrm{CO}}$ as $y$ is varied close to $y_{2}(k)$ 10, 11]. This hysteresis is associated with well-defined metastable phases of the model.

The main aim of this paper is to understand the dynamical response of the model near the discontinuous transition. We present an extensive finite-size scaling analysis of results from kinetic Monte Carlo simulations that indicates, more conclusively than previous studies, that the system undergoes a first-order nonequilibrium phase transition along a coexistence curve that terminates at a critical point. We calculate several points on the coexistence curve. Next we measure the lifetimes of the metastable phases associated with the decay from the low (high) CO coverage phase to the high (low) coverage phase. We find that the statistics of the metastable lifetimes are well described by the Kolmogorov-Johnson-MehlAvrami (KJMA) 13, 14, 15] theory of phase transformation by nucleation and growth near a first-order equilibrium phase transition.

The outline of the paper is as follows. In Sec. II we define the model and describe the Monte Carlo simulation techniques used. In Sec. [II] we present and discuss the numerical results obtained: in Sec. IIA we show how we calculate the coexistence curve and present a finite-size scaling analysis of the fluctuations and of the fourth-order cumulant of the order parameter; in Sec. [IIB we present the measurements of the lifetimes of the metastable states associated with the transition and show how their behavior is described by the KJMA theory. Our conclusions are summarized in Sec. IV]

\section{MODEL AND SIMULATION}

The ZGB model with desorption is simulated on a square lattice of linear size $L$ that represents the catalytic surface. A Monte Carlo simulation generates a sequence of trials: $\mathrm{CO}$ or $\mathrm{O}_{2}$ adsorption with probability $1-k$ and $\mathrm{CO}$ desorption with probability $k$. In the case of adsorption a $\mathrm{CO}$ or $\mathrm{O}_{2}$ molecule is selected with probability $y$ and $1-y$ respectively 


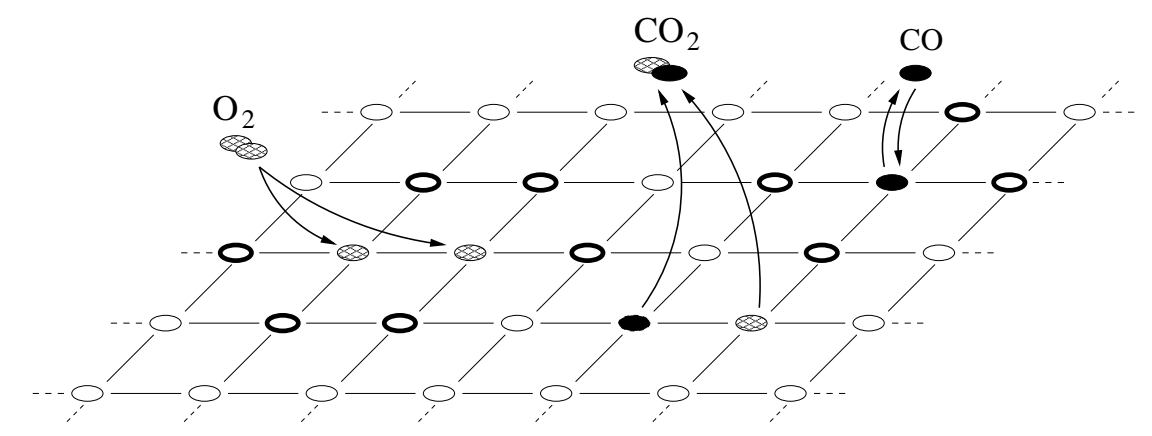

FIG. 1: Schematic representation of the algorithm. See discussion in the text.

3, 11]. These probabilities are the relative impingement rates of both molecules and are proportional to their partial pressures. The algorithm works in the following way. A site $i$ is selected at random. In the case of desorption, if $i$ is occupied by $\mathrm{CO}$ the site is vacated and the trial ends, if not the trial also ends. In the case of adsorption, if a CO molecule is selected it can be adsorbed at the empty site $i$ if none of its nearest neighbors are occupied by an $\mathrm{O}$ atom. Otherwise, one of the occupied $\mathrm{O}$ neighbors is selected at random and removed from the surface, leaving $i$ and the selected neighbor vacant. This move simulates the $\mathrm{CO}+\mathrm{O}$ $\rightarrow \mathrm{CO}_{2}$ surface reaction following the adsorption of $\mathrm{CO} \cdot \mathrm{O}_{2}$ molecules can be adsorbed only if a pair of nearest-neighbor sites are vacant. If the adsorbed molecule is selected to be $\mathrm{O}_{2}$ a nearest neighbor of $i, j$, is selected at random, and if it is occupied the trial ends. If both $i$ and $j$ are empty, the trial proceeds, and the $\mathrm{O}_{2}$ molecule is adsorbed and dissociates into two $\mathrm{O}$ atoms. If none of the remaining neighbors of $i$ is occupied by a $\mathrm{CO}$ molecule, one $\mathrm{O}$ atom is located at $i$, and if none of the neighbors of $j$ is occupied by a CO molecule, then the other $\mathrm{O}$ is located at $j$. If any neighbors of $i$ are occupied by a CO, then one is selected at random to react with the $\mathrm{O}$ at $i$ such that both sites are vacated. The same reaction happens at site $j$ if any of its neighbors are filled with a $\mathrm{CO}$ molecule. This process mimics the $\mathrm{CO}+\mathrm{O} \rightarrow \mathrm{CO}_{2}$ surface reaction following $\mathrm{O}_{2}$ adsorption. A schematic representation of this algorithm is shown in Fig. 1. We emphasize that the ZGB model, both with and without $\mathrm{CO}$ desorption, is an intrinsically nonequilibrium model that is fully defined by these dynamic rules. In contrast to systems considered in equilibrium thermodynamics, its properties are not derived from a Hamiltonian. We shall return to this point in Sec. IIB

For our simulations we assume periodic boundary conditions. The time unit is one Monte Carlo Step per Site (MCSS), in which each site, on average, is visited once. For 


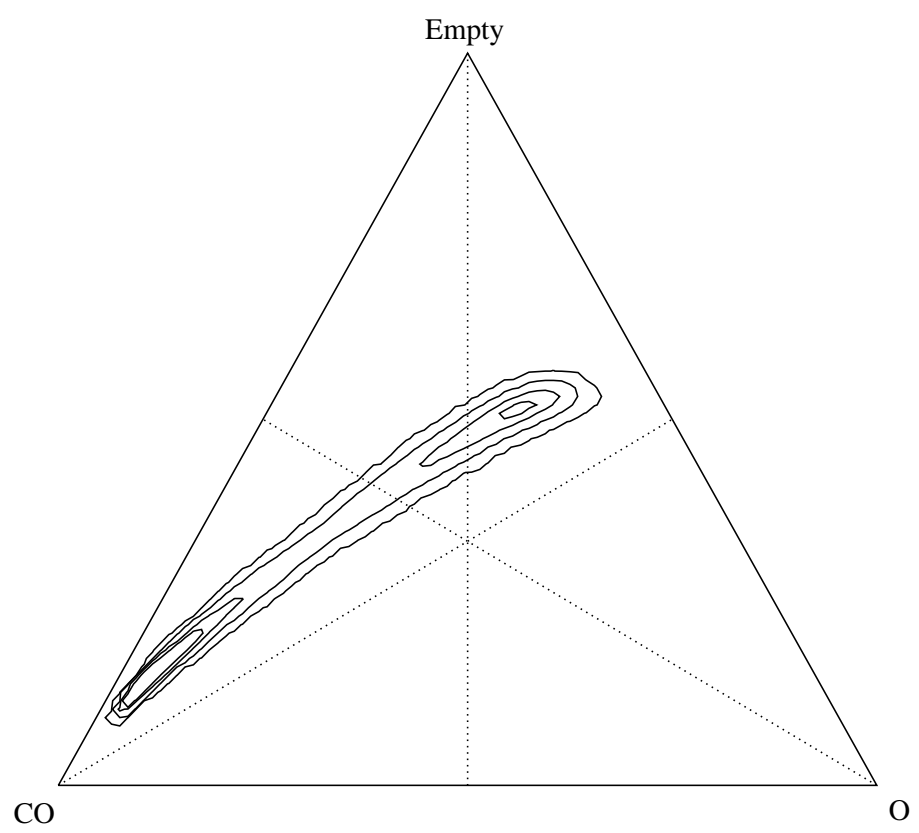

FIG. 2: Contour plot of the projection of $10^{6}$ MCSS of simulation onto the ternary phase diagram for $k=0.02, y=0.5332$, and $L=100$.

measurements of stationary quantities, the system was allowed to reach stationarity before data were recorded for analysis. Averages were taken over $10^{3}$ independent simulation runs.

\section{RESULTS}

We use a standard ternary phase diagram to plot the fraction of sites occupied by CO molecules: the $\mathrm{CO}$ coverage, $\theta_{\mathrm{CO}}$; the $\mathrm{O}$ coverage, $\theta_{\mathrm{O}}$, and the fraction of empty sites, $\theta_{\mathrm{E}}$. In Fig. 2 we present a contour plot of a histogram based on the projection of $10^{6}$ MCSS onto the plane of the phase diagram. For the chosen parameters and observation times the system undergoes several transitions between the low and high CO coverage phases. From the fact that the set of phase points is nearly parallel to the line $\theta_{\mathrm{O}} / \theta_{\mathrm{E}}=1 / 2$, it is evident that the $\mathrm{CO}$ coverage gives more information about the kinetic phase transitions than $\theta_{\mathrm{O}}$ or $\theta_{\mathrm{E}}$. 


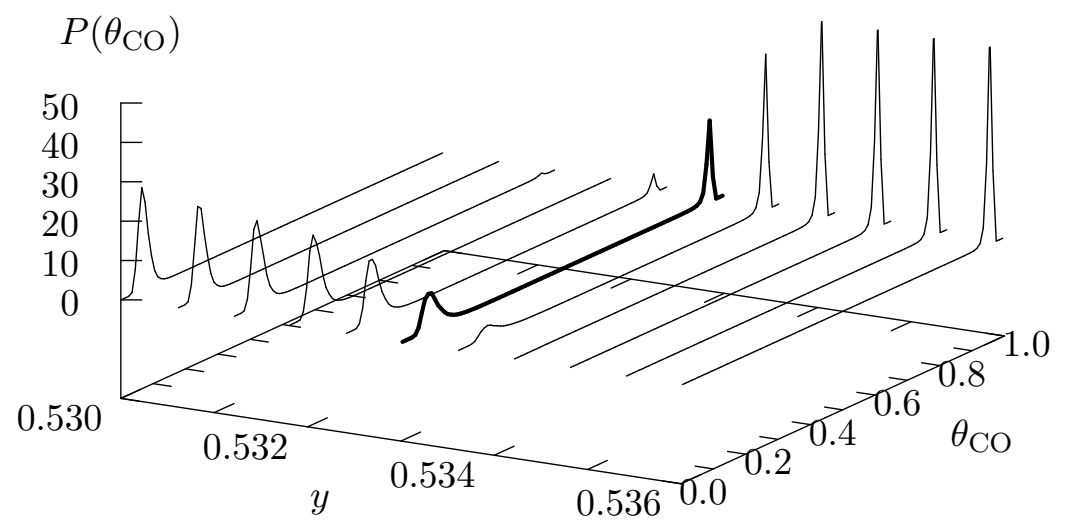

FIG. 3: Order-parameter probability distribution, $P\left(\theta_{\mathrm{CO}}\right)$, shown vs $y$ for $k=0.03$ and $L=100$. The distribution for the value of $y$ closest to the coexistence value is shown with a bold line.

\section{A. Determination of the Coexistence Curve}

We estimate $P\left(\theta_{\mathrm{CO}}\right)$, the probability distribution for $\theta_{\mathrm{CO}}$, by recording the number of times $N_{i}$ that the coverage fell in the intervals $[0, \epsilon),[\epsilon, 2 \epsilon), \ldots,[1-\epsilon, 1](\epsilon=0.01)$, such that $\sum_{i} N_{i}=N$ is the total number of MCSS. Then, the probability that $\theta_{\mathrm{CO}}$ has a value in the interval $[(i-1) \epsilon, i \epsilon)$ is $P_{i}=N_{i} / N \epsilon$, such that

$$
\int_{0}^{1} P\left(\theta_{\mathrm{CO}}\right) d \theta_{\mathrm{CO}}=\sum_{i} P_{i} \epsilon=1 .
$$

In Fig. 3] we show $P\left(\theta_{\mathrm{CO}}\right)$ versus $y$. In the regions (below and above $y_{2}$ ) where the histograms are unimodal, the system consists of one single phase. For a very narrow range of $y$, the histograms are bimodal, indicating two distinct phases. At the coexistence point $y_{2}(k)$, the areas under both peaks are equal [16, 17].

We define a measure of the fluctuations in $\theta_{\mathrm{CO}}$ in a $L \times L$ system in the standard way as

$$
X_{L}=L^{2}\left(\left\langle\theta_{\mathrm{CO}}^{2}\right\rangle_{L}-\left\langle\theta_{\mathrm{CO}}\right\rangle_{L}^{2}\right)
$$

where

$$
\left\langle\theta_{\mathrm{CO}}^{n}\right\rangle_{L}=\int_{0}^{1} \theta_{\mathrm{CO}}^{n} P\left(\theta_{\mathrm{CO}}\right) d \theta_{\mathrm{CO}}
$$




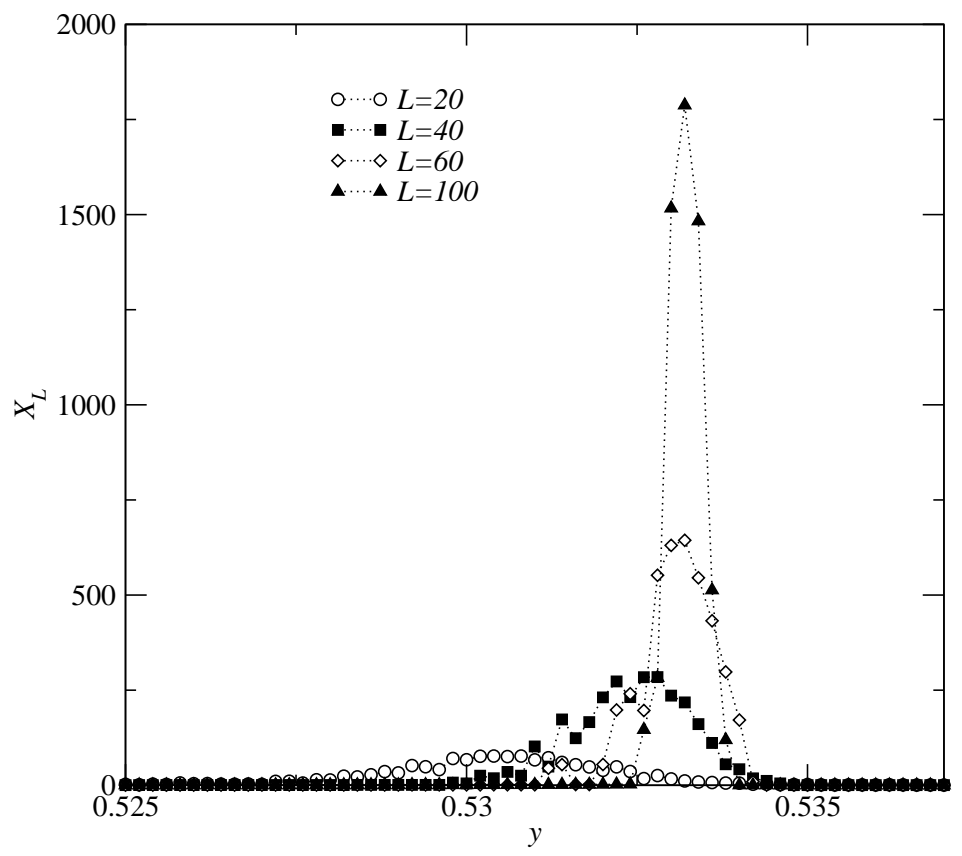

FIG. 4: The order-parameter fluctuation measure $X_{L}$, shown vs $y$ for $k=0.02$ and four system sizes. The dotted lines are guides to the eye. The values of $X_{L}$ have an error of approximately $5 \%$.

We measure $X_{L}$ as a function of $y$ for a fixed value of $k$ and several values of $L$. At a first-order equilibrium phase transition, the order-parameter fluctuations increase with the system size, such that the maximum value of $X_{L} \sim L^{d}$, where $d$ is the spatial dimension of the system [17, 18, 19, 20]. We will take the same scaling behavior to indicate a nonequilibrium first-order transition.

In Fig. 4 we show $X_{L}$ vs $y$ for four system sizes at $k=0.02$. For the four values of $L$ used, $X_{L}$ displays a clear peak, which moves and increases in height with increasing $L$.

In Fig. 5(a) we plot $\ln \left(X_{L}^{\max }\right)$ versus $\ln (L)$ for several values of $k$. A linear fit indicates a power-law divergence with $L$, such that the maximum value scales as $X_{L}^{\max } \sim L^{d^{\prime}}$ with $d^{\prime}=$ $1.96 \pm 0.02,1.91 \pm 0.05$, and $1.58 \pm 0.04$ for $k=0.02,0.03$, and 0.04 , respectively. A different method to extract the power-law exponent, which has some advantage in eliminating the effects of a nonsingular background term (as in $X_{L}=f+g L^{d^{\prime}}$ with $f$ and $g$ constants), is to consider

$$
\ln \left[\frac{X_{b L}^{\max }}{X_{L}^{\max }}\right] / \ln b=d^{\prime}+\mathcal{O}(1 / \ln b)
$$

with $L$ fixed at a relatively small value (here, $L=20$ ), and $b>1$. For large $L$ and $b$, the correction term is proportional to $f /(g \ln b)$, so that the exponent can be estimated 

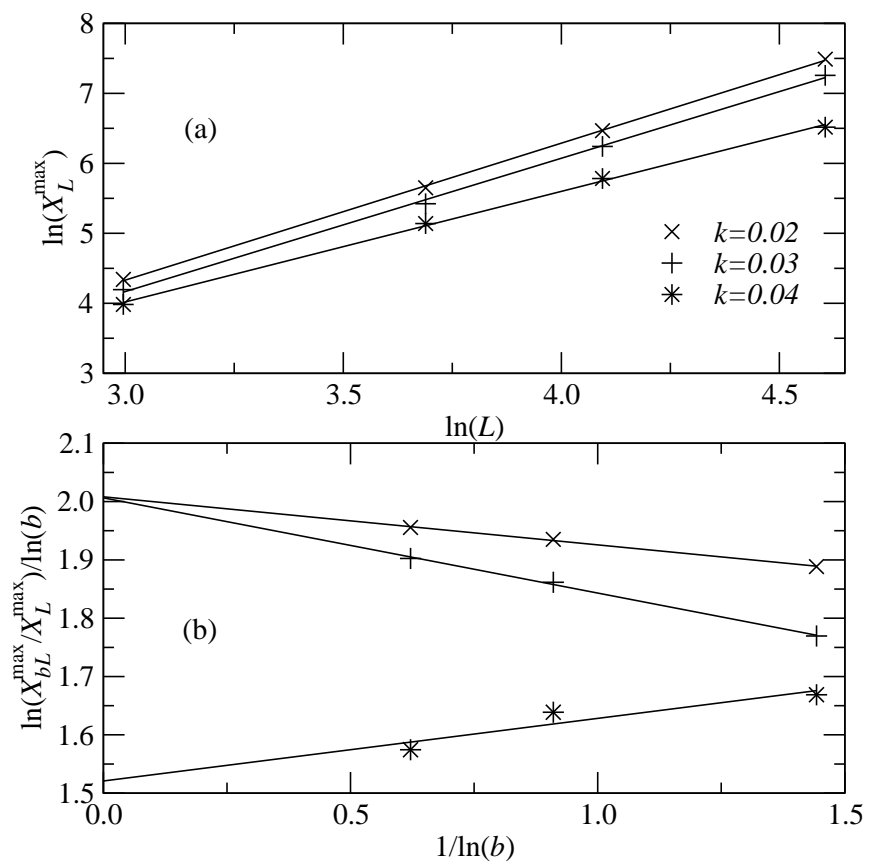

FIG. 5: (a) Plot of $\ln \left(X_{L}^{\max }\right)$ vs $\ln (L)$ and (b) plot of $\ln \left(X_{b L}^{\max } / X_{L}^{\max }\right) / \ln (b)$ with $L=20$ vs $1 / \ln (b)$, both for several values of $k$ and including all four system sizes. $X_{L}^{\max }$ is the maximum value of $X_{L}$, taken from figures similar to Fig. 4. The straight lines are the best linear fits to the data and give $X_{L}^{\max } \sim L^{d^{\prime}}$ with (a) $d^{\prime}=1.96 \pm 0.02,1.91 \pm 0.05$, and $1.58 \pm 0.04$; and (b) $d^{\prime}=2.008 \pm 0.005,2.006 \pm 0.008$, and $1.52 \pm 0.04$; for $k=0.02,0.03$, and 0.04 , respectively.

by plotting the left-hand-side of Eq. (41) vs $1 / \ln b$ and extrapolating to $1 / \ln b=0$, as in Fig. 5(b). The resulting estimates are $d^{\prime}=2.008 \pm 0.005,2.006 \pm 0.008$, and $1.52 \pm 0.04$ for $k=0.02,0.03$, and 0.04 , respectively.

These results indicate that the system undergoes a first-order phase transition, $d^{\prime} \approx$ $d=2$, at $y=y_{2}(k)$, generating a coexistence curve that terminates at a critical point $0.03<k_{c}<0.04$. A previous estimate based on a study of the fractal dimension of the interface between the two phases gives $0.039<k_{c}<0.04$ [9]. Another study, which estimates $k_{c}(L)$ as the value of $k$ where the double-peaked histograms become single-peaked, gives $k_{c}(L \rightarrow \infty)=0.04060$ [1]]. However, from the known two-peaked shape of the orderparameter distribution at the equilibrium Ising critical point [21], we believe that this method should yield a slight overestimate of $k_{c}$. Reference [11] also reports preliminary results on the fourth-order cumulant of the $\mathrm{CO}$ coverage that are consistent with an Ising-like critical point at $k_{c}=0.0406$. However, the cumulants were calculated only for very small lattice 
sizes $(L=10,20$, and 40$)$ and do not constitute a definite proof of the location of the critical point, as the authors duly point out [1]]. Below we present a similar cumulant study, but with larger lattice sizes.

We also calculated the relation between the value of the probability distribution $P\left(\theta_{\mathrm{CO}}\right)$ at either of the peaks of the bimodal distribution, $P_{\max }$, and its value at the local minimum between the peaks, $P_{\text {min }}$. For a first-order equilibrium phase transition these quantities satisfy the relation

$$
\frac{P_{\max }}{P_{\min }} \propto \exp (c L)
$$

where $c$ is proportional to the equilibrium interface tension between the two phases. If the relation is applied to the present system, we would expect $c$ to be positive and decrease with increasing $k$. Our results indicate that $c(k)$ is positive only for $k<0.03$, suggesting that $0.02<k_{c}<0.03$. These results corroborate again that the system has a first-order phase transition for small $k$. However, they suggest a much lower value for $k_{c}$ than indicated by our other techniques and the previous results by others [9, 11]. We find this result quite interesting and believe it may be due to several reasons. Most obvious is the significant difficulty in locating and measuring the peaks, which are extremely narrow in $y$. Perhaps more significant is the fact that this non-Hamiltonian nonequilibrium system does not possess a well-defined surface tension that could be associated with the parameter $c$ in Eq. (5). This point will be further discussed in Sec. IIIB, where we also show that the cluster interfaces in this system are much rougher than in conventional Hamiltonian systems. As a result, we do not consider the method for determining $k_{c}$, based on Eq. (5), very accurate. The fact that different techniques give different results are an indication of the difficulties associated with locating $k_{c}$ in this model, even for relatively large systems.

A useful tool for detecting phase transitions in simulations of finite equilibrium systems is the fourth-order reduced cumulant of the order parameter [17, 19, 20]. For $\theta_{\mathrm{CO}}$ it takes the form

$$
u_{L}=1-\frac{\mu_{4}}{3 \mu_{2}^{2}}
$$

where,

$$
\mu_{n}=\left\langle\left(\theta_{\mathrm{CO}}-\left\langle\theta_{\mathrm{CO}}\right\rangle\right)^{n}\right\rangle=\int_{0}^{1}\left(\theta_{\mathrm{CO}}-\left\langle\theta_{\mathrm{CO}}\right\rangle\right)^{n} P\left(\theta_{\mathrm{CO}}\right) d \theta_{\mathrm{CO}},
$$

is the $n$th central moment of the CO coverage. The equal-area bimodal distribution corresponding to coexistence yields a positive maximum for the cumulant vs $y$, flanked on either 

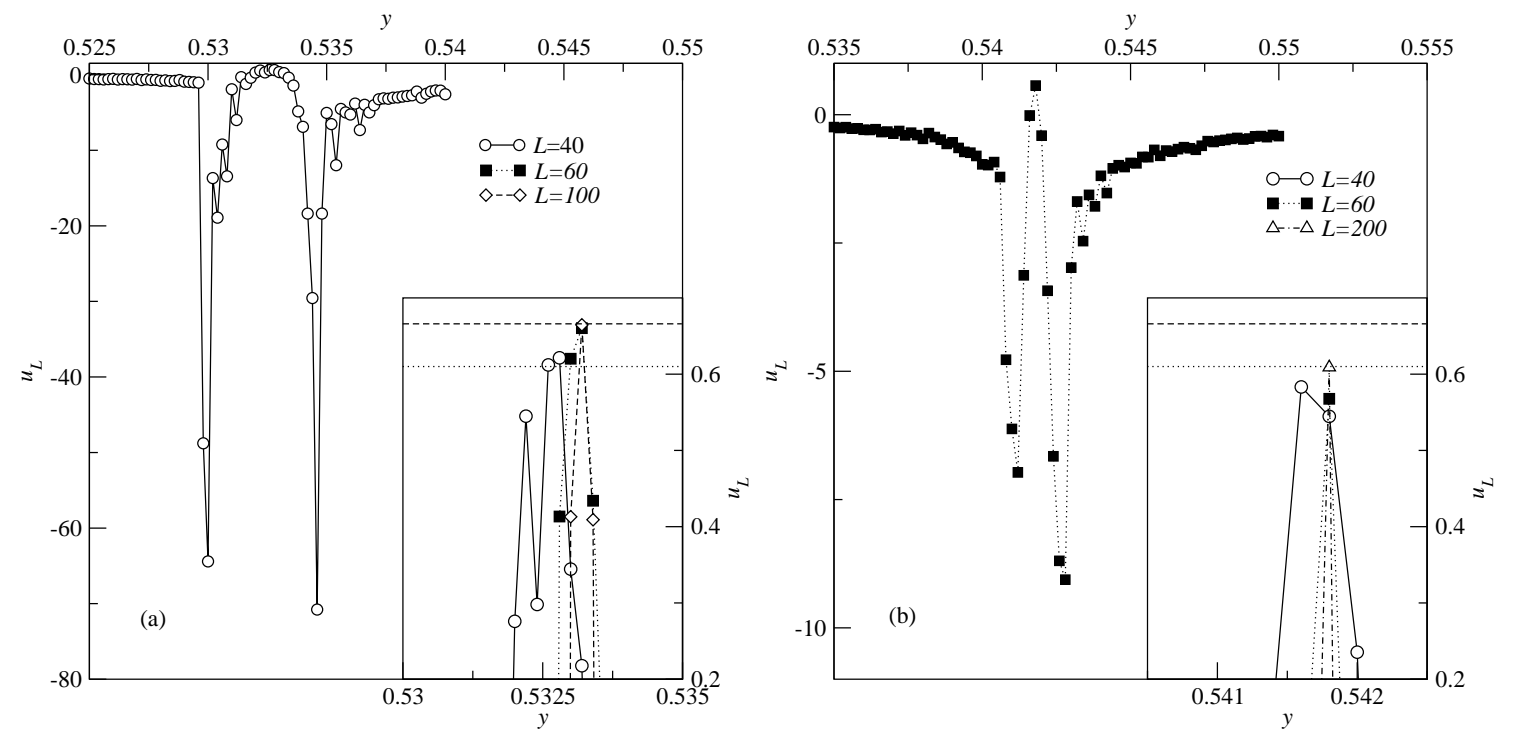

FIG. 6: Dependence of the fourth-order reduced cumulant $u_{L}$ on $y$, for (a) $k=0.02$ and (b) $k=0.04$. The $P\left(\theta_{\mathrm{CO}}\right)$ histograms indicate that the minima of $u_{L}$ correspond to the transitions from unimodal to bimodal distribution. The maximum between them gives the coexistence point $y_{2}(k, L)$. The horizontal lines in the insets correspond to $u_{L}=2 / 3$ (dashed) and $u_{\text {Ising }}^{*} \approx 0.61$ (dotted).

side by negative minima and approaching zero far away from the transition. Since the cumulant essentially is a tool to determine the shape of the order-parameter distribution, it can also be used for nonequilibrium phase transitions, such as the one studied here. The maxima of $u_{L}$ define the $L$-dependent coexistence line, $y_{2}(k, L)$. In Fig. [6 we show the dependence of $u_{L}$ on $y$ for several values of $L$ and for $k=0.02$ and $k=0.04$, respectively. When $k=0.02$, the maximum value of $u_{L}$ is very close to $2 / 3$, however when $k=0.04$ the maximum is very close to 0.61 , consistent with the proximity of an Ising-like critical point.

The finite-size scaling theory of equilibrium first-order phase transitions implies that the shift in the position of the transition in a finite system of linear size $L$ with periodic boundary conditions is inversely proportional to the system volume, $L^{d}$ [18, 20, 22] (here the dimension $d=2$ ). Although there is no analogous scaling theory for the present nonequilibrium system, we here attempt to use the same scaling relation,

$$
y_{2}(k, L)-y_{2}(k) \propto L^{-2}
$$

where $y_{2}(k)$ is the transition value of the $\mathrm{CO}$ adsorption rate $y$ in the infinite- $L$ limit. In Fig. 7 we plot $y_{2}(L)$ vs $1 / L^{2}$ for $L=20,40,60,80,100,200$, and 300 for several values 


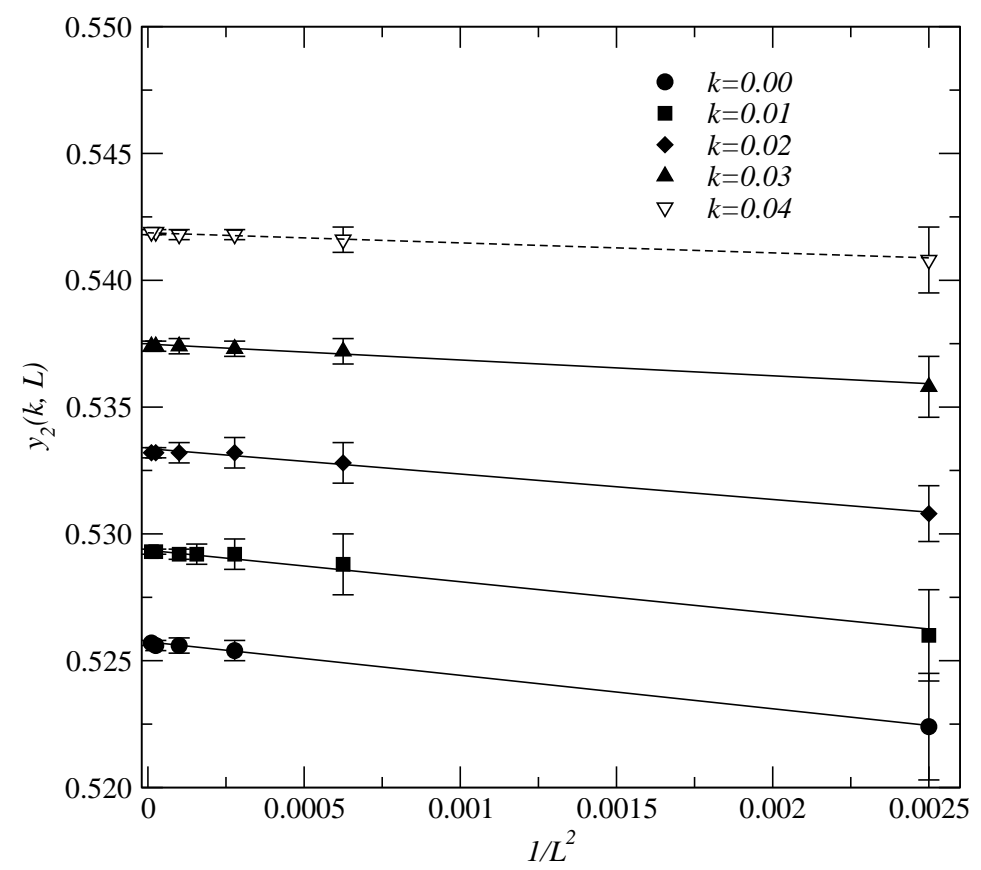

FIG. 7: Critical CO adsorption rate $y_{2}(k, L)$, shown vs $1 / L^{2}$ for several values of the desorption rate $k$. The straight lines are weighted least-squares fits, yielding the estimated $y_{2}(k)$ for $L=\infty$.

of $k$. The error bars are calculated as the half width of the peaks of the $u_{L}$ vs $y$ curves 19, 20]. As seen from the figure, the points fall very close to the straight line representing a weighted least-squares fit, yielding a good estimate (within $10^{-2} \%$ ) of $y_{2}(k)$ for each $k$ when $L \rightarrow \infty$. Our result $y_{2}=0.5257(3)$ for $k=0$ is consistent with previous studies that give $y_{2}=0.52560(1)$ [12] and $y_{2}=0.52583(9)$ [23] for $L \rightarrow \infty$.

In Fig. 8 we show several points on the coexistence curve between the low and high $\mathrm{CO}$ coverage phases. The coexistence curve is almost linear with only a slight curvature. By extrapolating a quadratic fit of the data to $k=0.04060$ we obtain $y_{2}=0.542(3)$ in agreement with the previous result $y_{2}=0.54212(10)$ for $k_{c}$ 11].

\section{B. Metastability}

In this section we calculate the time associated with the decay from the low (high) $\mathrm{CO}$ coverage phase to the high (low) CO coverage phase, $\tau_{\mathrm{p}}\left(\tau_{\mathrm{d}}\right)$, and determine its dependence on the $\mathrm{CO}$ pressure and the system size. In order to do this, we prepare the system with initial pressure $y=y_{\mathrm{w}}$ such that $y_{1}<y_{\mathrm{w}}<y_{2}(k)\left(y_{\mathrm{w}}>y_{2}(k)\right)$ and then suddenly change $y$, such that $y>y_{2}(k)\left(y<y_{2}(k)\right)$. Then the initial low (high)- coverage phase becomes 


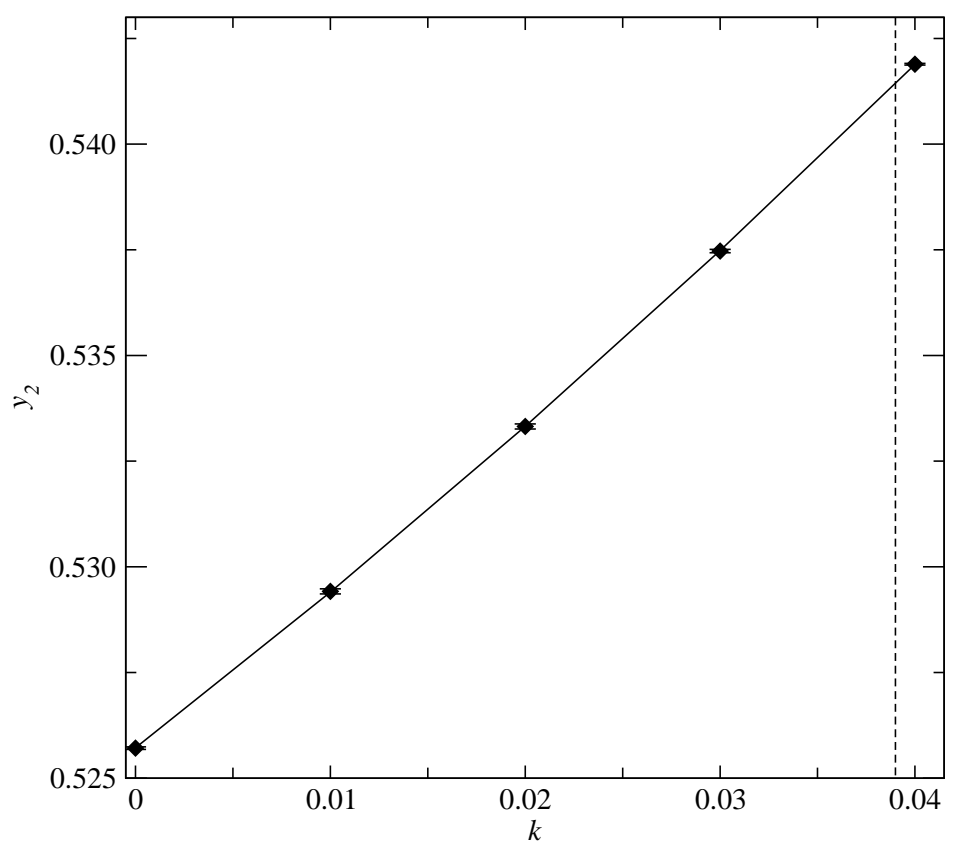

FIG. 8: Some points of the coexistence curve, analogous to the pressure vs temperature phase diagram for a fluid in equilibrium. The continuous line represents a quadratic fit to the data. The vertical dashed line indicates the estimate $k_{c} \approx 0.039$ from Ref. [9].

metastable and eventually decays to the high (low)-coverage phase. The system is considered to have left the metastable phase once its coverage reaches a certain cutoff value $\theta_{\mathrm{CO}}^{*}$. To avoid that recrossing events back to the metastable phase are mistaken for decay events, the cut-off is selected such that it is not too close to the metastable coverage value. The statistics of the decay times are analyzed for $n=500$ independent runs.

Since the value of $y_{\mathrm{w}}$ that determines how far the initial system is from the transition point $y_{2}(k)$ is somewhat arbitrary, it is necessary to evaluate how the decay times depend on it. Figure 9(a) indicates that, in the region of interest, the average decay time from the low to the high CO coverage phase, $\left\langle\tau_{\mathrm{p}}\right\rangle$, while being dependent on the final pressure $y$, is fairly independent of the pressure at which the system is prepared, $y_{\mathrm{w}}$. In the following we then take $y_{\mathrm{w}}=0.45$ to calculate $\tau_{\mathrm{p}}$. It is also necessary to evaluate the dependence of $\left\langle\tau_{\mathrm{p}}\right\rangle$ on the selected cut-off value $\theta_{\mathrm{CO}}^{*}$. In Fig. 9(b) we plot $\left\langle\tau_{\mathrm{p}}\right\rangle$ vs $\theta_{\mathrm{CO}}^{*}$ for different values of $k$ and $y$. Clearly, $\left\langle\tau_{\mathrm{p}}\right\rangle$ increases with $\theta_{\mathrm{CO}}^{*}$, however there is a region where it is relatively weakly dependent on the cutoff. We choose to perform our measurements of $\left\langle\tau_{\mathrm{p}}\right\rangle$ at $\theta_{\mathrm{CO}}^{*}=0.65$, well inside this region.

Figure 10 indicates that the average decay times associated with the decontamination 

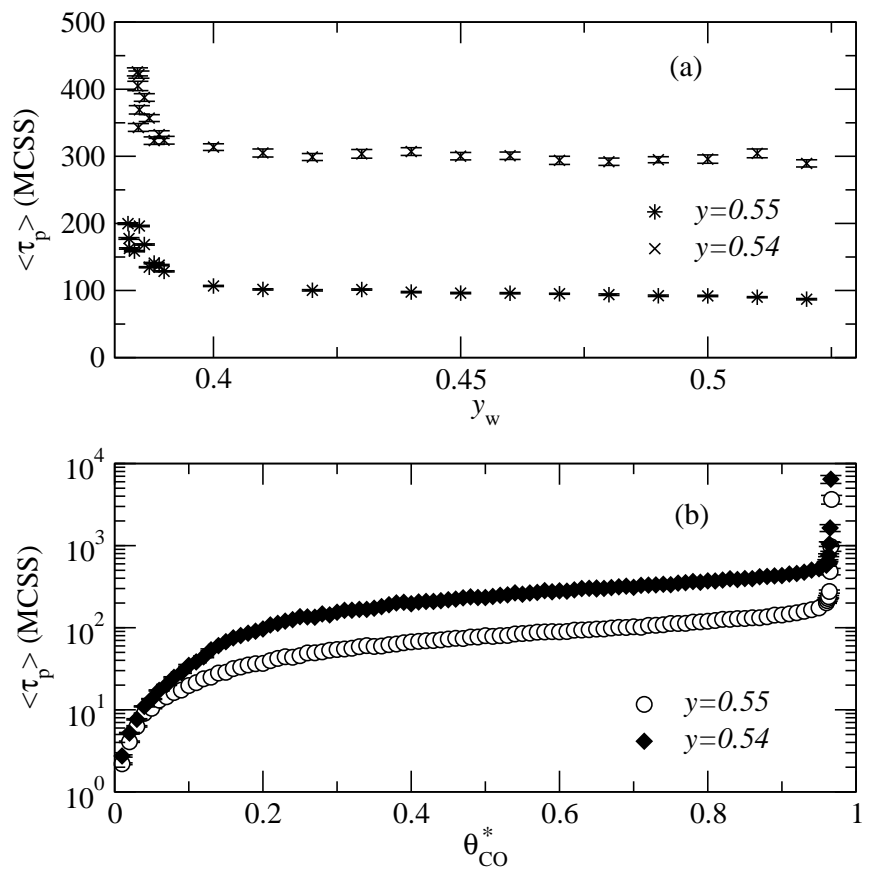

FIG. 9: $\left\langle\tau_{\mathrm{p}}\right\rangle$ as a function of (a) $y_{\mathrm{w}}$ with $\theta_{\mathrm{CO}}^{*}=0.65$, and (b) $\theta_{\mathrm{CO}}^{*}$ with $y_{\mathrm{w}}=0.475$; for $k=0.02$, $L=100$ and two different values of $y$.

of the CO surface, $\left\langle\tau_{\mathrm{d}}\right\rangle$, i.e. the relaxation time from the high CO coverage phase to the low-coverage phase, behaves in a similar way to $\left\langle\tau_{\mathrm{p}}\right\rangle$. Figure 10(a) clearly indicates that, to an even higher degree than in the poisoning process, $\left\langle\tau_{\mathrm{d}}\right\rangle$ is independent of $y_{\mathrm{w}}$. We choose $y_{\mathrm{w}}=0.57$ for our calculations. Figure 10(b) indicates that, as expected, $\left\langle\tau_{\mathrm{d}}\right\rangle$ increases as $\theta_{\mathrm{CO}}^{*}$ decreases, but there is a range of values of the cut-off where the dependence is relatively small. In the following we calculate $\left\langle\tau_{\mathrm{d}}\right\rangle$ with $\theta_{\mathrm{CO}}^{*}=0.45$, which lies in this region.

We define the quantity

$$
\Delta=\left|y-y_{2}\right|
$$

which measures how far the system is from the coexistence curve and depends on $k$ and $L$ through $y_{2}$. In Figs. 11 and 12 we present snapshot configurations obtained during the relaxation from the low to the high coverage phase and from the high to the low coverage phase, respectively, when $\Delta^{-1}$ is small, i.e., far from the transition. In Figs. 13 and 14 we show snapshot configurations for a much larger value of $\Delta^{-1}$, i.e., close to the transition. The difference in the decay mechanisms is evident from the figures. Figures 11 and 12 clearly suggest that when $\Delta^{-1}$ is small, the system decays by nucleation and growth of multiple droplets of the stable phase. In contrast, Figs. 13 and 14 show that when $\Delta^{-1}$ is large, 

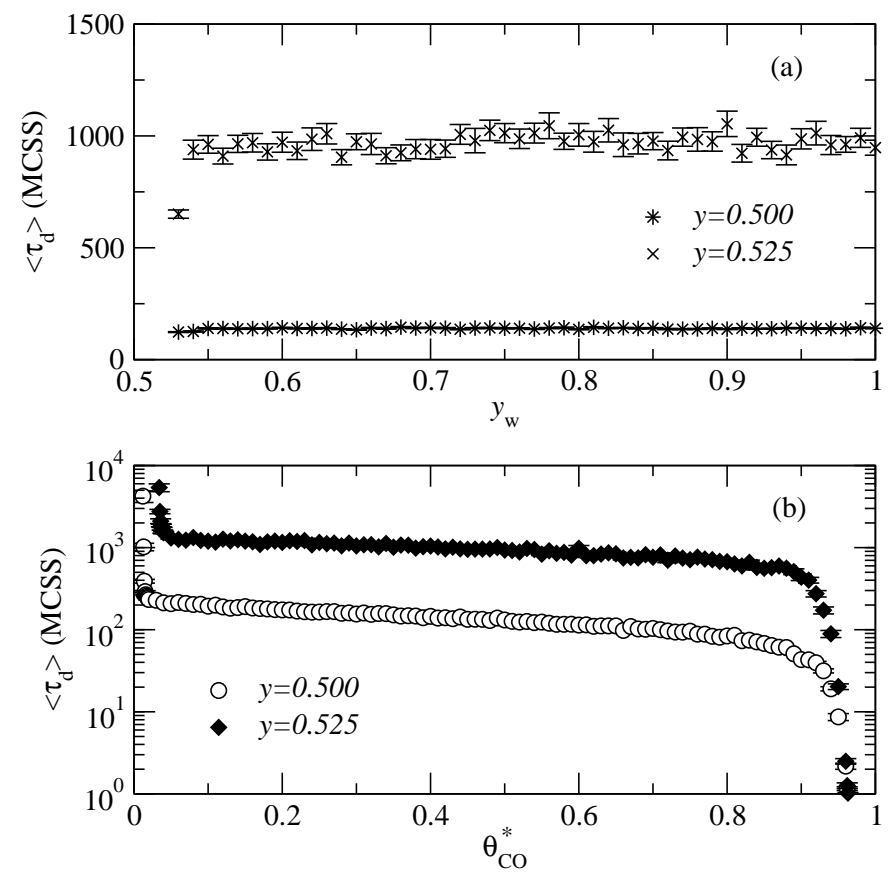

FIG. 10: $\left\langle\tau_{\mathrm{d}}\right\rangle$ as a function of (a) $y_{\mathrm{w}}$ with $\theta_{\mathrm{CO}}^{*}=0.45$, and (b) $\theta_{\mathrm{CO}}^{*}$ with $y_{\mathrm{w}}=0.57$; for $k=0.02$, $L=100$ and two different values of $y$.

the system decays by nucleation and growth of a single droplet of the stable phase, which eventually takes over the entire system. The probability distributions of $\tau_{\mathrm{p}}$ and $\tau_{\mathrm{d}}$, shown in Figs. 15] and 16, respectively, also indicate clear differences between the statistics of the decay times near and far from the coexistence line. Far from coexistence $\left(\Delta^{-1}\right.$ small $)$, the decay times follow an approximately Gaussian distribution. In contrast, near the transition $\left(\Delta^{-1}\right.$ large $)$, the distribution is approximately exponential.

The statistics of the metastable lifetimes in the model studied here are strikingly similar to those found in Hamiltonian systems that decay toward thermodynamic equilibrium from a metastable phase associated with an equilibrium phase transition. Well-studied examples are metastable decay in kinetic Ising [24, 25, 26, 27] and lattice-gas models [28, 29] with such applications as magnetism switching and submonolayer adsorption. In the present paper we will for simplicity refer to this latter case as the "Hamiltonian" case, thus emphasizing the lack of a Hamiltonian and the consequent lack of a concept of thermodynamic equilibrium for the system studied in the present paper. The metastable decay in such a Hamiltonian system occurs via nucleation and subsequent growth of "droplets" inside which the order parameter is close to its equilibrium value, and it is well described by the classic KJMA 


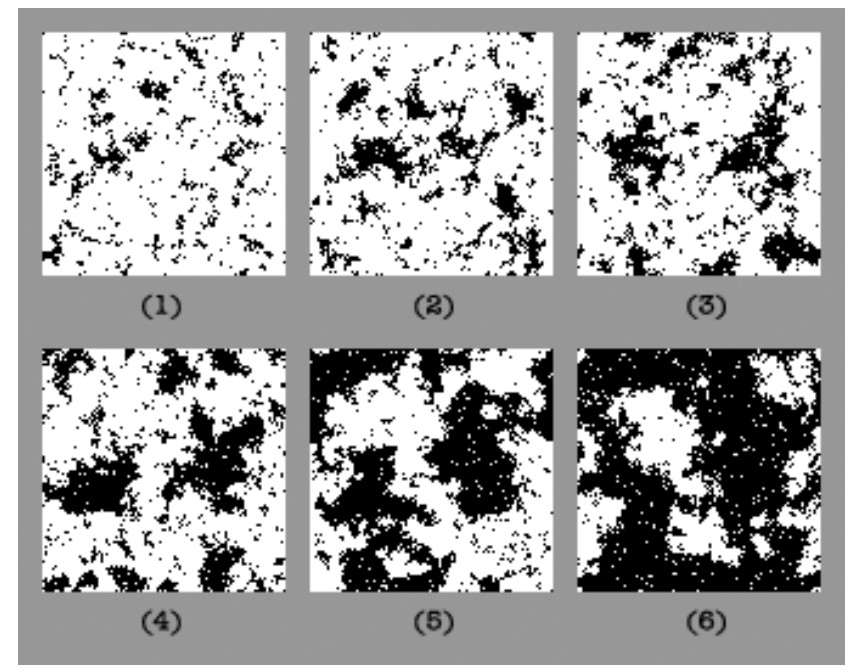

FIG. 11: Snapshot configurations obtained at different (unevenly spaced) times, after a sudden change of $y$ from $y_{\mathrm{w}}=0.45$ to $y=0.538>y_{2}(k)$, i.e., $\Delta^{-1} \approx 200$. For $k=0.02$ and $L=100$. Here and in Figs. 12 13, and 14, the black dots represent lattice sites occupied by CO, while both adsorbed $\mathrm{O}$ atoms and empty lattice sites are white.

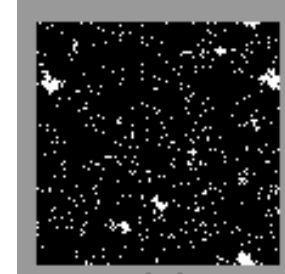

(1)

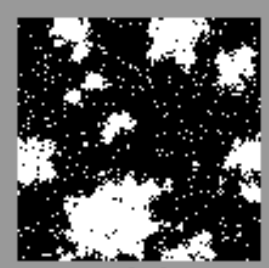

(4)

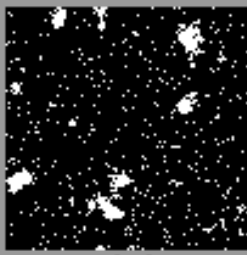

(2)

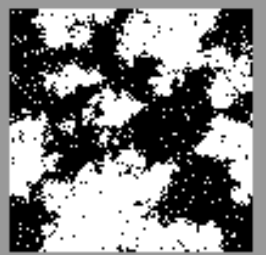

(5)

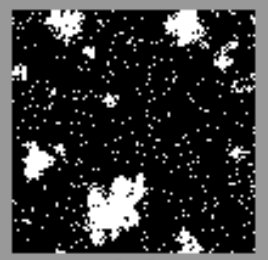

(3)

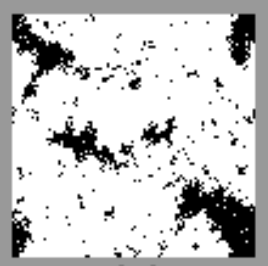

(6)

FIG. 12: Snapshot configurations obtained at different (unevenly spaced) times, after a sudden change of $y$ from $y=0.65=y_{\mathrm{w}}$ to $y=0.5232<y_{2}(k)$, i.e., $\Delta^{-1} \approx 100$. For $k=0.02$ and $L=100$. theory of phase transformation [13, 14, 15]. The basic assumption of this theory is that droplets of the stable phase nucleate in a Poisson process at a rate $I$ per unit volume. After nucleation, the droplet radius is assumed to grow with a constant speed, $v$. If the first droplet to nucleate grows fast enough to fill the system before another is likely to nucleate, 


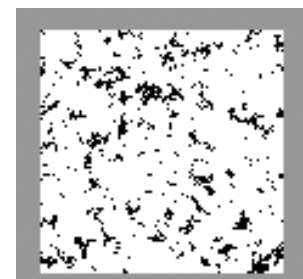

(1)

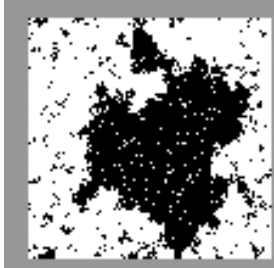

(4)

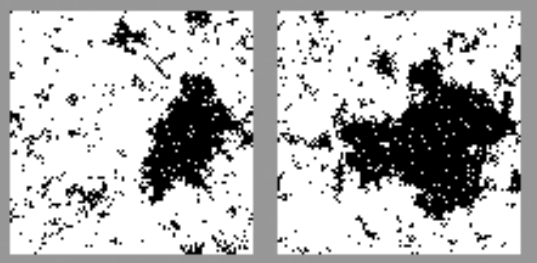

(2)

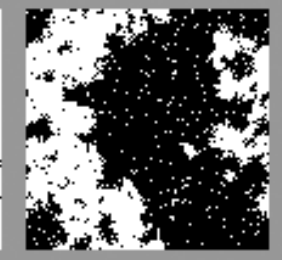

(5)
(3)

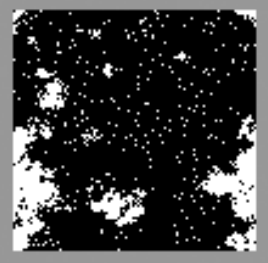

(6)

FIG. 13: Snapshot configurations obtained at different (unevenly spaced) times, after a sudden change of $y$ from $y=0.45=y_{\mathrm{w}}$ to $y=0.5338>y_{2}(k)$, i.e., $\Delta^{-1} \approx 2000$. For $k=0.02$ and $L=100$.

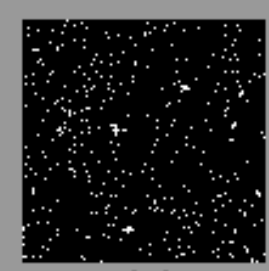

(1)

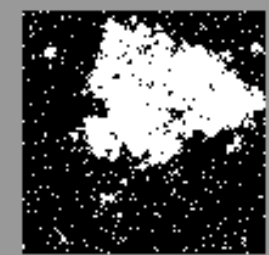

(4)

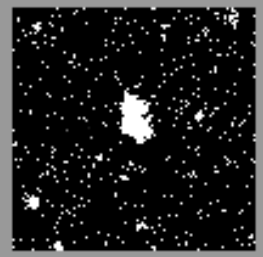

(2)

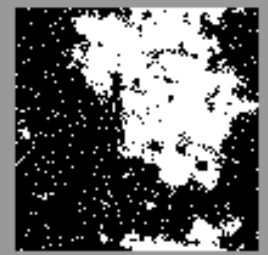

(5)

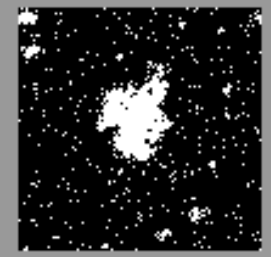

(3)

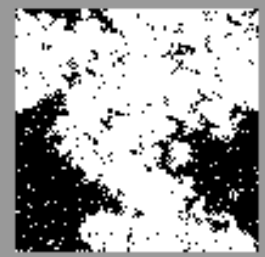

(6)

FIG. 14: Snapshot configurations obtained at different (unevenly spaced) times, after a sudden change of $y$ from $y=0.65=y_{\mathrm{w}}$ to $y=0.5315<y_{2}(k)$, i.e., $\Delta^{-1} \approx 500$. For $k=0.02$ and $L=100$.

then it completes the phase transformation by itself - a process known as single-droplet (SD) decay. However, if the growth is slow, so that many droplets can nucleate within the time it would take a single droplet to fill the system, the phase transformation will proceed via a large number of droplets that nucleate and grow in parallel - a process known as multidroplet (MD) decay. This simple observation can be turned into a formal scaling argument by constructing the characteristic length $R_{0}=(v / I)^{1 / 3}$, which is a measure of the 

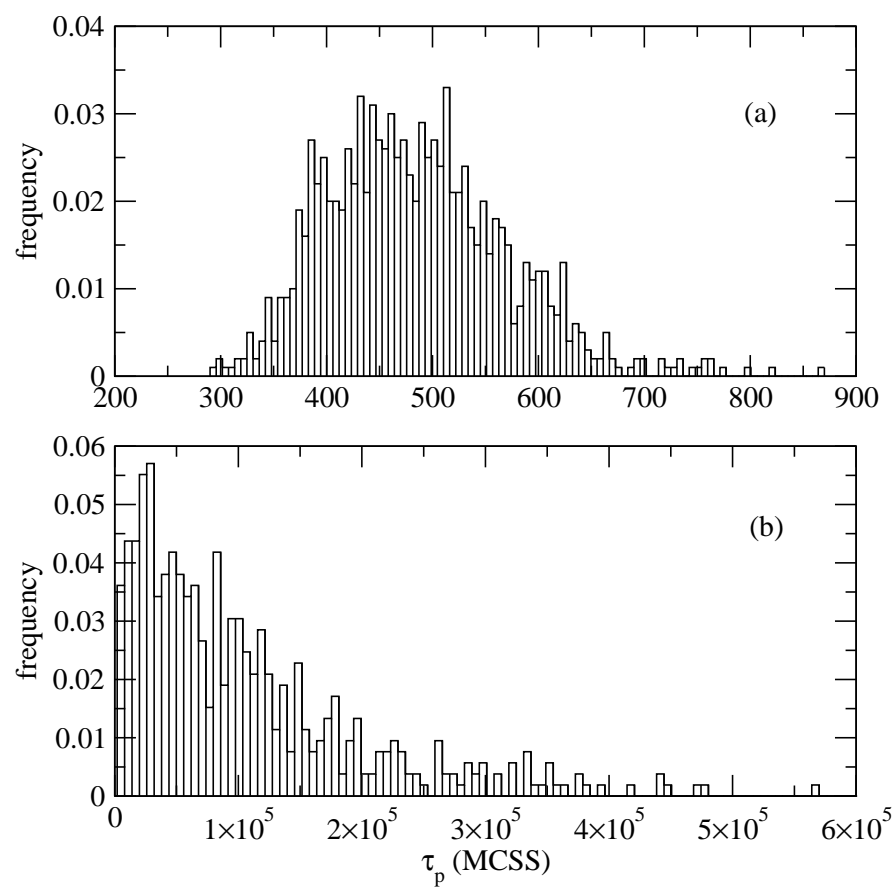

FIG. 15: Plot of distributions of $\tau_{\mathrm{p}}$ for $k=0.02$ and $L=100$, (a) $\Delta^{-1} \approx 200$ and (b) $\Delta^{-1} \approx 2000$. Note the very different time scales in (a) and (b).
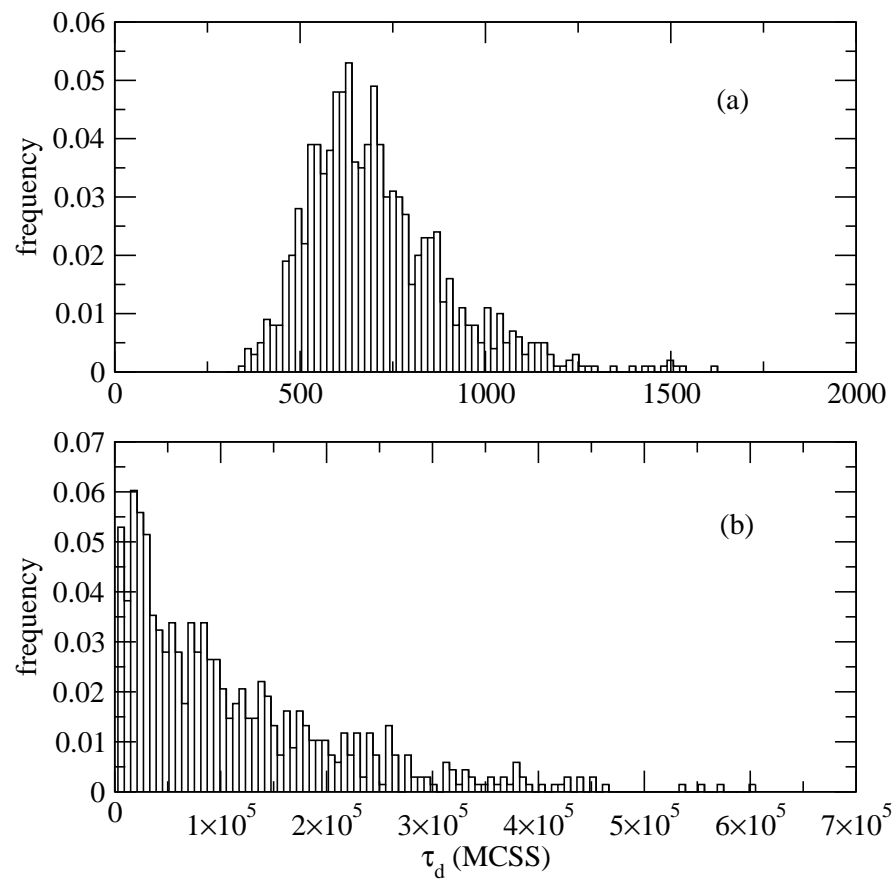

FIG. 16: Plot of distributions of $\tau_{\mathrm{d}}$ for $k=0.02$ and $L=100,(\mathrm{a}) \Delta^{-1} \approx 100$ and (b) $\Delta^{-1} \approx 500$. Note the very different time scales in (a) and (b). 
average size to which a droplet will grow before it touches another droplet. (Results are here reviewed only for $d=2$. Results for general $d$ can be found in, e.g., Refs. [24, 26] and references therein.) If $R_{0} \gg L$, the system is in the SD regime, and the metastable lifetime is simply the average time between nucleation events, $\tau_{\mathrm{SD}} \approx 1 /\left(I L^{2}\right)$. Since the nucleation events constitute a Poisson process, $\tau_{\mathrm{SD}}$ follows an exponential distribution, similar to the ones shown in Figs. 15(b) and 16(b) for the system discussed here. If, on the other hand, $R_{0} \ll L$, then the system is in the MD regime, and the metastable lifetime is obtained as $\tau_{\mathrm{MD}}=R_{0} / v=1 /\left(v^{2} I\right)^{1 / 3}$. Since the metastable decay in this case consists of a large number of droplets that nucleate and grow independently, $\tau_{\mathrm{MD}}$ follows a Gaussian distribution with a standard deviation proportional to $R_{0} / L$, similar to the ones shown in Figs. 15(a) and 16(a) for the system discussed here.

The SD regime with its exponential lifetime distribution is a subregion of a broader stochastic regime, while the MD regime with its narrow Gaussian distribution is part of a broader deterministic regime. The relative standard deviation of the lifetimes is defined by

$$
r=\frac{\sqrt{\left\langle\tau^{2}\right\rangle-\langle\tau\rangle^{2}}}{\langle\tau\rangle} \approx\left\{\begin{array}{ll}
R_{0} / L<1 & \text { in MD regime } \\
1 & \text { in SD regime }
\end{array} .\right.
$$

The limit between the SD and MD regimes is called the Dynamic Spinodal (DSP) and corresponds to $R_{0} \approx L$. It is, however, easier to estimate it as given by the values of $I$ and $v$ that yield $r=1 / 2[24]$.

So far, the KJMA results discussed do not require a specific dependence of the nucleation rate $I$ and growth velocity $v$ on the macroscopic control parameters, which for metastable decay in Hamiltonian systems are the applied magnetic field $H$ (or chemical potential or supersaturation for lattice-gas models) and the temperature $T$. In the present model the analogous quantities should be the distance from coexistence $\Delta$ and the desorption rate $k$. Hamiltonian systems are described by a free energy, and standard arguments of droplet theory show that for not too strong fields, $I \sim \exp [-c(T) /(T|H|)]$, where $c(T)$ is well approximated as proportional to the equilibrium interface tension between the metastable and equilibrium phases. For weak fields, $v \propto|H|$ - an effect that to a reasonable approximation can be ignored compared to the exponential dependence on $1 /|H|$ in $I$.

The present nonequilibrium system has no Hamiltonian and so no free-energy function. However, let us for the moment postulate that $I(\Delta, k) \sim \exp (-c(k) / \Delta)$ for reasonably small 

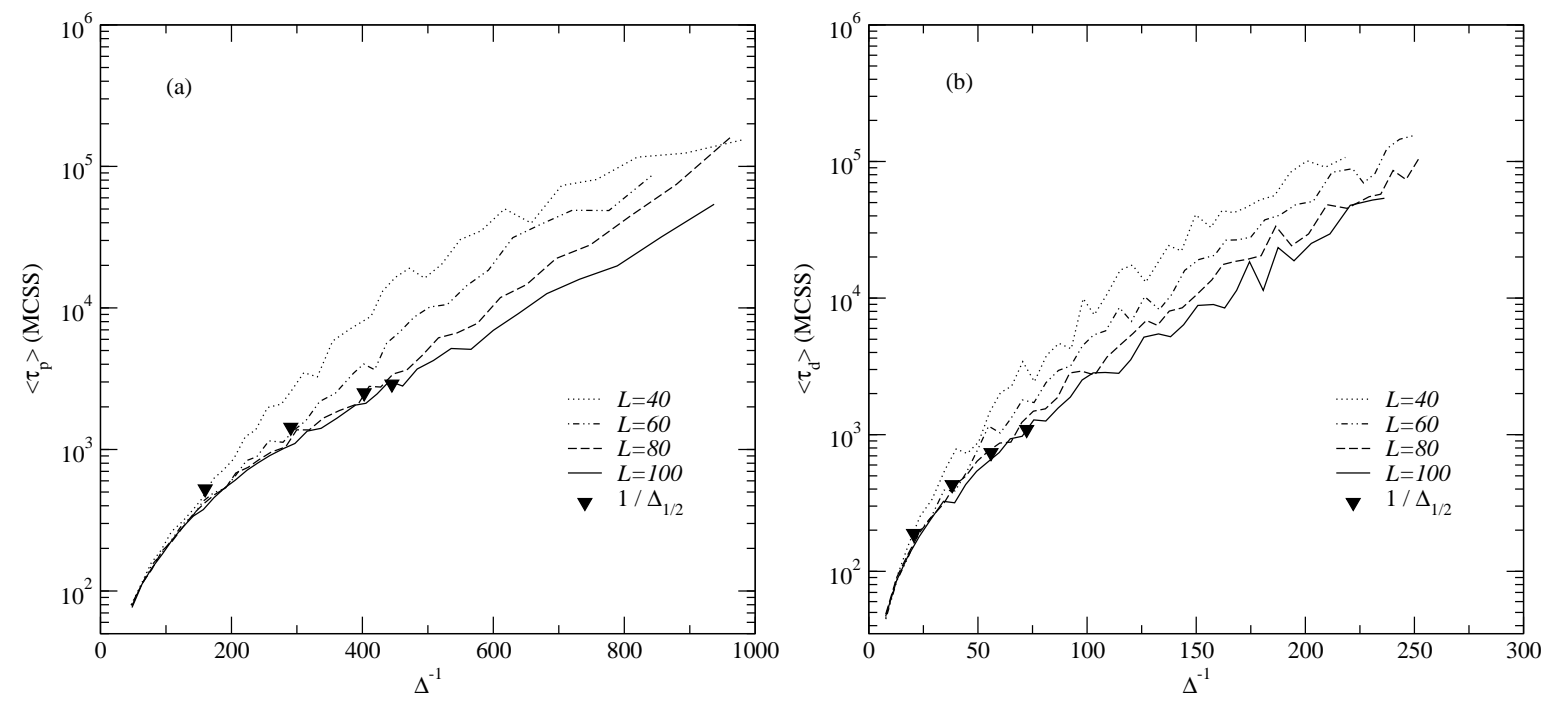

FIG. 17: Log-linear plot of (a) $\left\langle\tau_{\mathrm{p}}\right\rangle$ vs $\Delta^{-1}$ and (b) $\left\langle\tau_{\mathrm{d}}\right\rangle$ vs $\Delta^{-1}$, shown for $k=0.01$ and different values of $L$. The solid inverted triangles indicate $\Delta_{1 / 2}^{-1}$ for each value of $L$. Approximate data collapse is seen for $\Delta^{-1}<\Delta_{1 / 2}^{-1}$.

$\Delta$, and that $v(\Delta, k)$ depends comparatively weakly on its parameters so that it can be taken as approximately constant. Following the method of data analysis introduced in Ref. [24], we then expect logarithmic plots of $\left\langle\tau_{\mathrm{p}}\right\rangle$ and $\left\langle\tau_{\mathrm{d}}\right\rangle$ (and of $r$ in the MD regime) vs $1 / \Delta$ to be approximately linear. Furthermore, the general KJMA arguments given above indicate that the lifetimes should be independent of $L$ in the MD regime and $\propto L^{-2}$ in the SD regime.

Figure [17 shows log-linear plots of $\left\langle\tau_{\mathrm{p}}\right\rangle$ and $\left\langle\tau_{\mathrm{d}}\right\rangle$ vs $\Delta^{-1}$ for $k=0.01$ and different sizes. Similar plots were also obtained for $k=0.02$ (not shown). The plots clearly indicate that there is a regime, corresponding to small $\Delta^{-1}$, where $\left\langle\tau_{\mathrm{p}}\right\rangle$ is independent of $L$. Figure 18 strongly indicates that when $\Delta^{-1}$ is large, the decay times are inversely proportional to $1 / L^{2}$. Only the data for $L=40$ do not seem to follow this dependence. We believe it is possible that $L=40$ becomes smaller than the critical droplet size for large $\Delta^{-1}$ (incipient "coexistence regime," see Ref. [24]).

To further explore the applicability of the KJMA theory and our postulate to the model, we next determine the dynamic spinodal, $\Delta_{\mathrm{DSP}}$, that separates the stochastic and the deterministic regimes. We calculate the relative standard deviation $r$ of Eq. (10), which is shown on a logarithmic scale vs $\Delta^{-1}$ in Fig. 19, where error bars are estimated by standard 

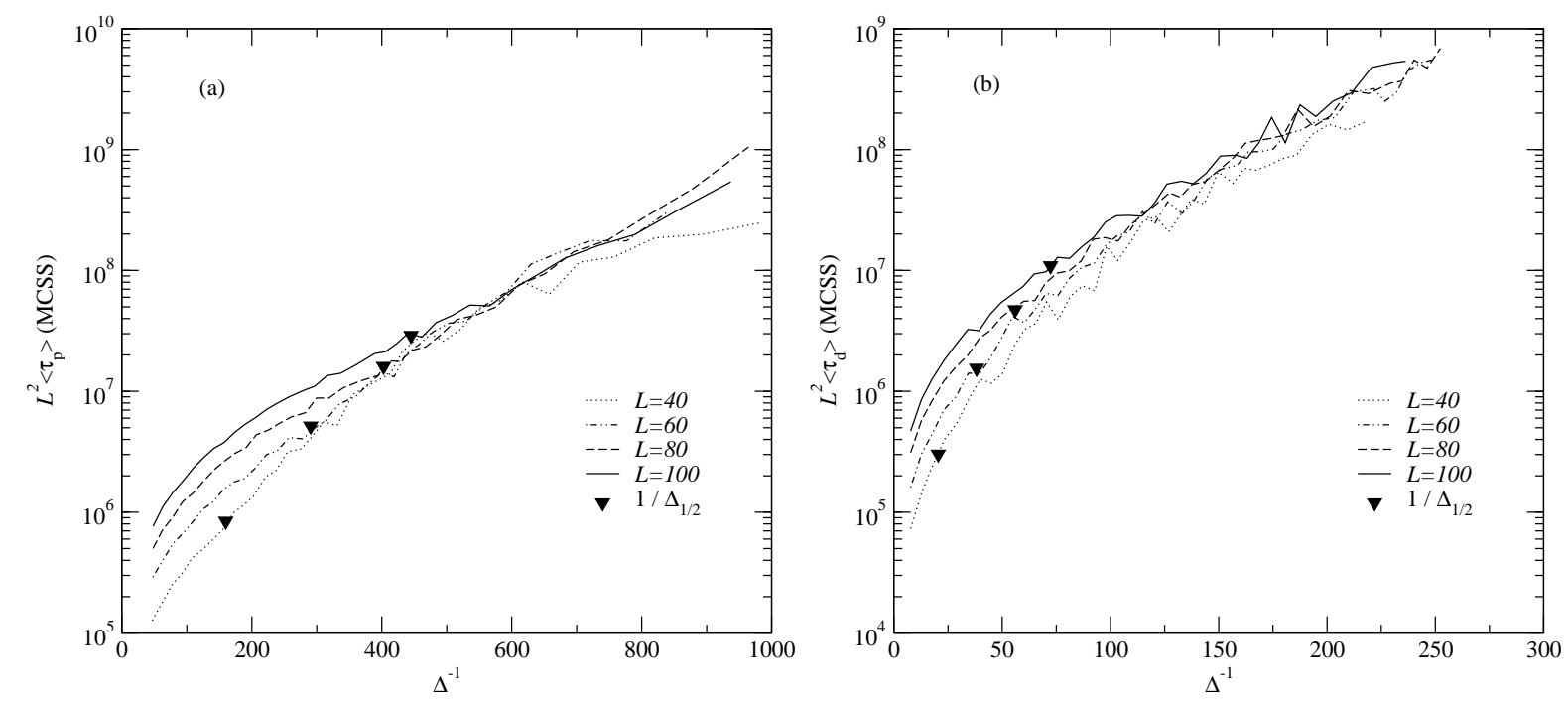

FIG. 18: Log-linear plot of (a) $L^{2}\left\langle\tau_{\mathrm{p}}\right\rangle$ vs $\Delta^{-1}$ and (b) $L^{2}\left\langle\tau_{\mathrm{d}}\right\rangle$ vs $\Delta^{-1}$, shown for $k=0.01$ and different values of $L$. The solid inverted triangles indicate $\Delta_{1 / 2}^{-1}$ for each value of $L$. Approximate data collapse is seen for $\Delta^{-1}>\Delta_{1 / 2}^{-1}$.

error-propagation methods as

$$
\sigma_{r} \approx \frac{r}{\sqrt{n-1}}\left(1+\frac{n-1}{n} r^{2}\right)^{\frac{1}{2}} .
$$

As can be seen, $r$ crosses over from the approximately linear behavior expected from our postulate for small $\Delta^{-1}$ to $r \approx 1$ for larger $\Delta^{-1}$. We take as our estimate for $\Delta_{\text {DSP }}$ the value $\Delta_{1 / 2}$ for which $r=0.5$. This crossover is determined for each value of $L$ from the crossing of a weighted least-squares fit to $\ln r$ in the linear region of Fig. 19 with the horizontal line $r=0.5$. The resulting estimates are shown in Fig. 20 as $1 / \Delta_{1 / 2}$ vs $L$ on a logarithmic scale, with error bars estimated from those in Fig. [19 by standard error-propagation methods. The numerical results are consistent with the analytical prediction based on our postulate,

$$
\Delta_{\mathrm{DSP}} \sim 1 / \ln L
$$

The asymptotic $L$ dependence of the lifetime at the DSP, analogously given by

$$
\langle\tau\rangle \propto L / \Delta_{\mathrm{DSP}} \sim L(\ln L)
$$

is illustrated in Fig. 21, For each $L$ this lifetime was obtained by interpolation between the two closest field values bracketing $\Delta_{1 / 2}$, for which simulations had been performed. The 

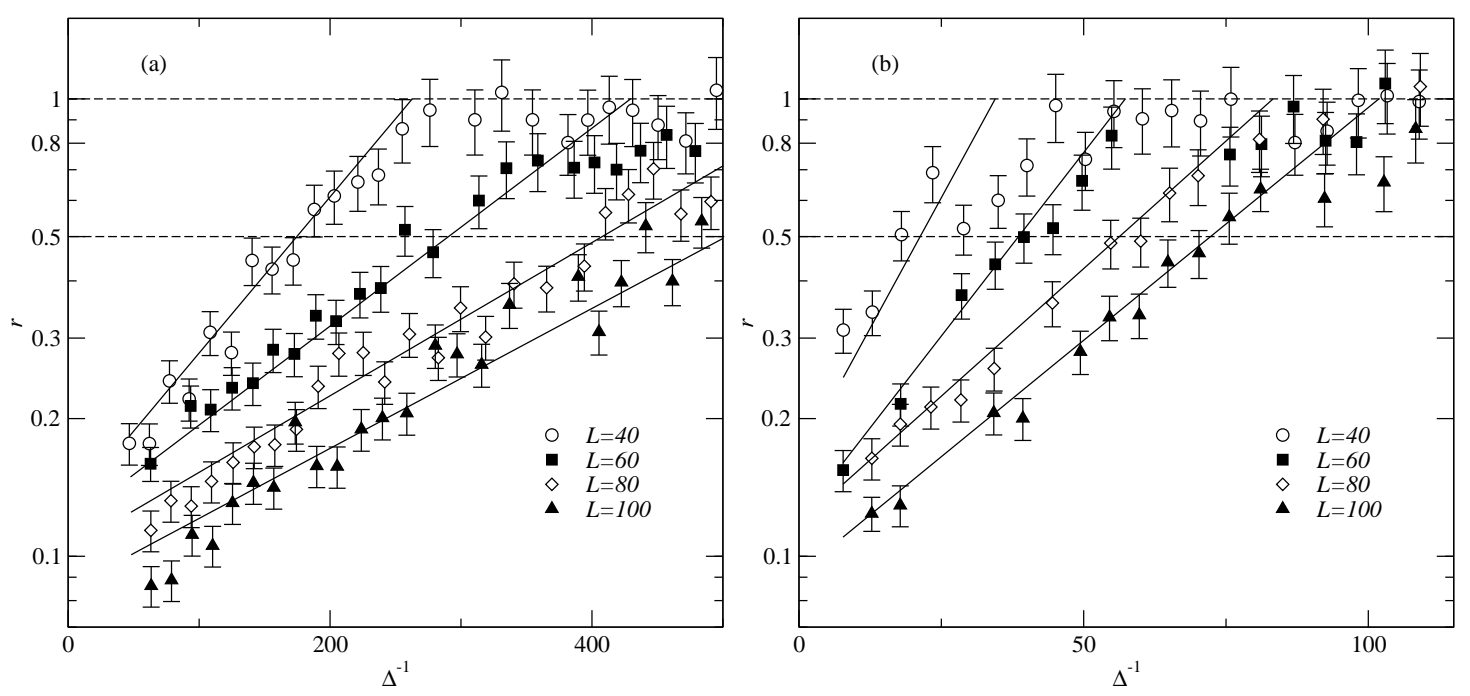

FIG. 19: The relative standard deviation $r$ of (a) $\left\langle\tau_{\mathrm{p}}\right\rangle$ and (b) $\left\langle\tau_{\mathrm{d}}\right\rangle$, shown on a logarithmic scale vs $\Delta^{-1}$ for $k=0.01$. The behavior of $r$ crosses over from the approximate straight line expected from our postulate in the deterministic regime to $r \approx 1$ in the stochastic regime. The solid lines are weighted least-squares fits to the data in the linear region.
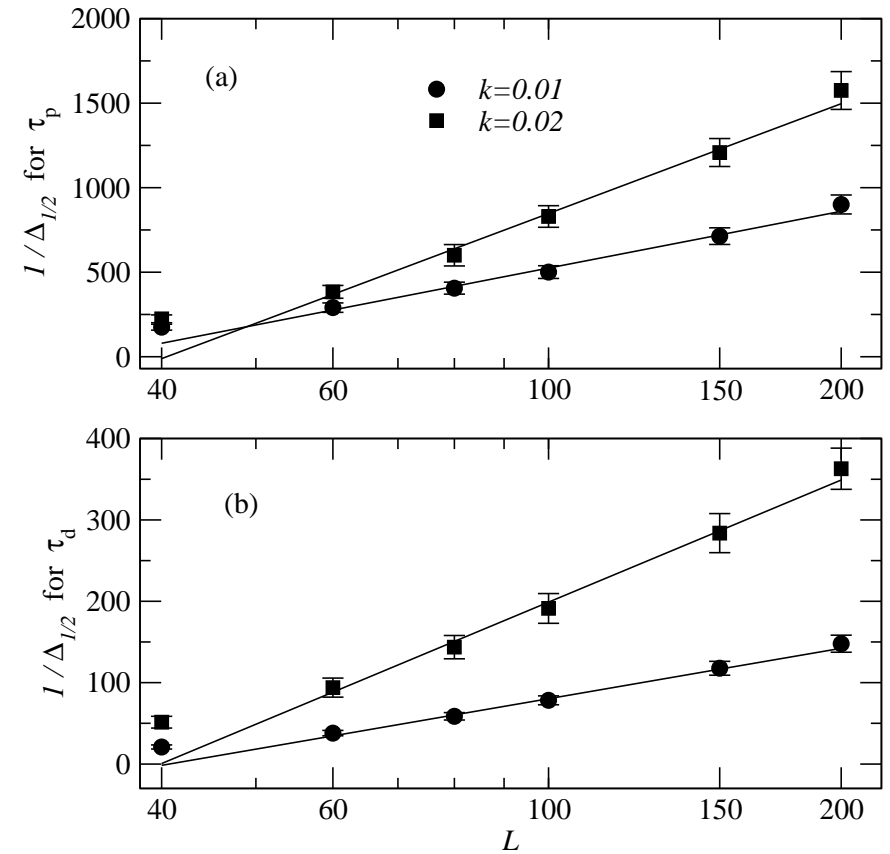

FIG. 20: The estimates $1 / \Delta_{1 / 2}$ for $1 / \Delta_{\text {DSP }}$ for (a) poisoning and (b) decontamination as obtained from Fig. 19 and analogous data, shown vs $L$ on a logarithmic scale. The solid lines are weighted least-squares fits excluding the points corresponding to $L=40$. 

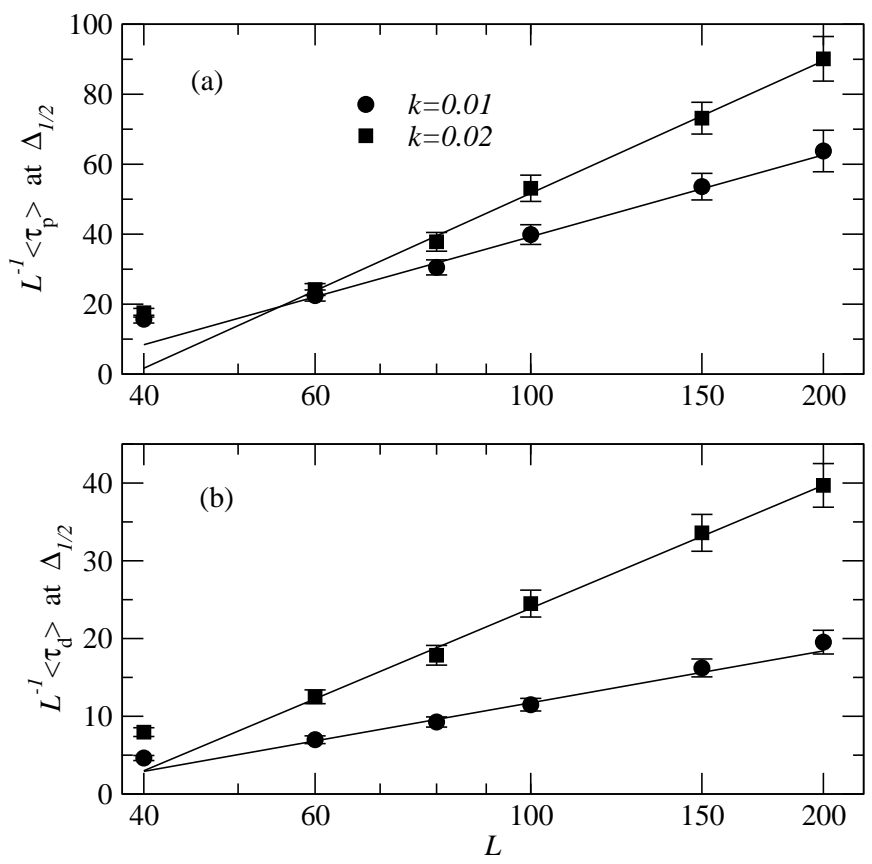

FIG. 21: (a) $\left\langle\tau_{\mathrm{p}}\right\rangle / L$ at $1 / \Delta_{1 / 2}$ and (b) $\left\langle\tau_{\mathrm{d}}\right\rangle / L$ at $1 / \Delta_{1 / 2}$, shown vs $L$ on a logarithmic scale. The solid straight lines are weighted least-squares fits. Excluding the points corresponding to $L=40$, the figures supports the asymptotic behavior $\left\langle\tau_{\mathrm{p}}\right\rangle \propto L \ln L$ at the DSP.

uncertainty in the resulting estimate was obtained by standard error propagation, taking the standard deviation in $\langle\tau\rangle$ from Eq. (10) with $r=1 / 2$, and $\langle\tau\rangle$ from Fig. 17.

While a direct analogue of the surface tension does not exist in the present system, the results described above strongly suggest that it obeys a decay mechanism very similar to the one described by the standard KJMA theory of phase transformation by nucleation and growth, which predicts well-defined single-droplet and multidroplet regimes. A significant difference between our Fig. 17 and analogous figures showing the metastable lifetime for a Hamiltonian system vs inverse field or supersaturation (see, e.g, Fig. 2 of Ref. 24] and Fig. 2 of Ref. [27]), is that we here see no marked change in the slope of the curves at the DPT. One possible explanation is that the "effective surface tension" in the present case may decrease substantially with increasing $\Delta$, in contrast to the situation in Hamiltonian systems.

The decay times increase as $y$ approaches the transition line $y_{2}(k)$, however their behaviors depend on the direction of approach to the transition value, as can be seen in Fig. 22. In a previous work we have shown how this asymmetry can be exploited to enhance the catalytic 


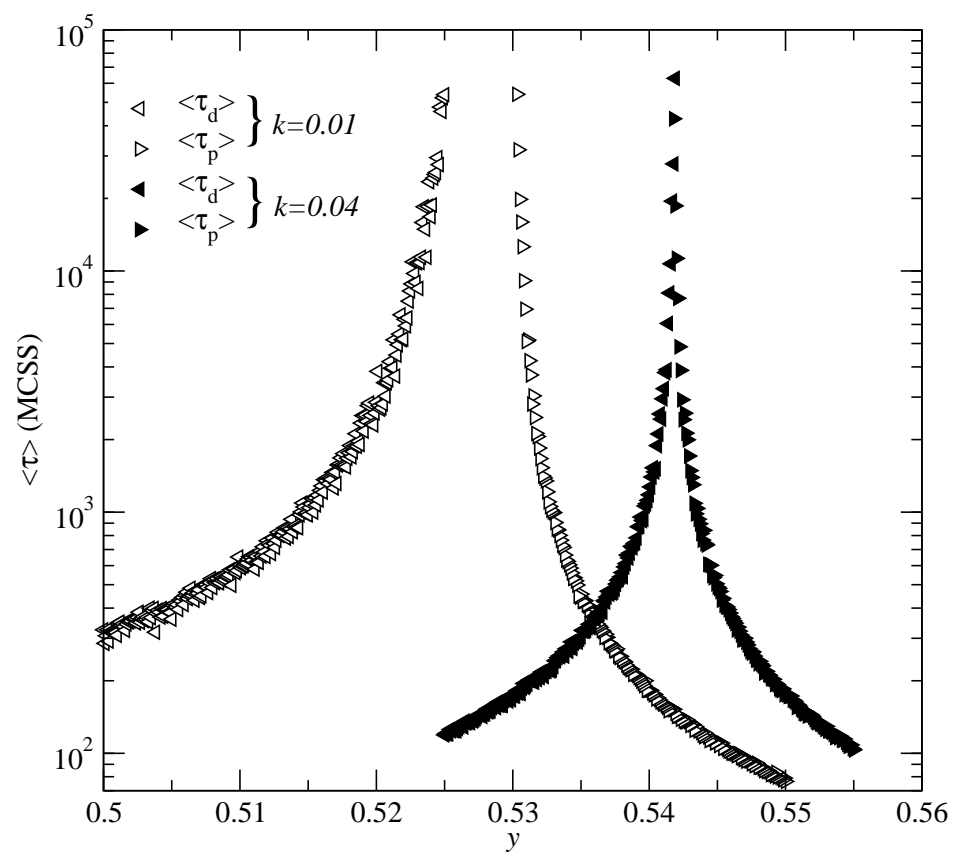

FIG. 22: Decay times as functions of $y$ when the system evolves toward the low CO coverage region $\left(\theta_{\mathrm{CO}}^{*}=0.45, y_{\mathrm{w}}=0.55\right.$, left-pointing triangles $)$ and when it evolves toward the high CO coverage region $\left(\theta_{\mathrm{CO}}^{*}=0.65, y_{\mathrm{w}}=0.475\right.$, right-pointing triangles) for $k=0.01$ and $k=0.04$, and $L=100$. As discussed in the text, the divergence is exponential in $1 /\left|y-y_{2}(k, L)\right|=1 / \Delta$.

activity by subjecting the system to periodic variation of the external pressure with periods related to the decay times in each direction [10].

The asymmetry between the decay times when the system evolves to the low CO coverage phase, $\left\langle\tau_{\mathrm{d}}\right\rangle$, and the decay time toward the high CO coverage phase, $\left\langle\tau_{\mathrm{p}}\right\rangle$, becomes more evident as the desorption parameter $k$ decreases, as can also be seen in Fig. 22. The extreme case occurs when $k=0$, where $\left\langle\tau_{\mathrm{d}}\right\rangle=\infty$, independently of the value of $y$, since in the high CO coverage phase the surface then becomes irreversibly poisoned with CO, whereas $\left\langle\tau_{\mathrm{p}}\right\rangle$ remains finite and dependent on $y$.

In Fig. 22 it can be seen that $\left\langle\tau_{\mathrm{d}}\right\rangle$ and $\left\langle\tau_{\mathrm{p}}\right\rangle$ appear to diverge at the same value of $y$. At this point the system spends, on average, the same amount of time in the low and high CO coverage phases, and it agrees with the value for the coexistence point $y_{2}$, calculated from the order-parameter distribution in Sec. IIIA. Since at this point the average decay time is of the order of $10^{4} \mathrm{MCSS}$, during the observation time of $10^{6}$ MCSS it is very probable that several transitions occur between the two phases. These transitions are observed in 
Fig. 3] where it can be seen that the probability distribution, $P\left(\theta_{C O}\right)$ is bimodal and quite symmetric, indicating that $\left\langle\tau_{\mathrm{d}}\right\rangle \approx\left\langle\tau_{\mathrm{p}}\right\rangle$ along the coexistence curve for finite $L$.

\section{CONCLUSIONS}

We have investigated by kinetic Monte Carlo simulation the dynamical behavior of a ZGB model with desorption near the coexistence curve between the active and the CO poisoned nonequilibrium phases. We perform an extensive finite-size scaling analysis of the fluctuations and of the fourth-order reduced cumulant of the $\mathrm{CO}$ coverage, which plays the role of an order parameter. Our results strongly indicate, as also previously suggested by others, that the system undergoes a first-order nonequilibrium phase transition between the active and the CO poisoned phases. The coexistence curve terminates at a critical value of the desorption rate. We also calculated several points on the coexistence curve.

Next we calculated the system-size dependence of the decay times of the metastable phases when the system is driven into the $\mathrm{CO}$ poisoned phase from the active phase, and vice versa. We found that near the coexistence curve the decay times are inversely proportional to $1 / L^{2}$, and the decay mechanism consists of the nucleation and growth of a single supercritical droplet of the stable phase. In contrast, far from the coexistence curve, the decay times are independent of the system size, and the decay proceeds by random nucleation of many droplets of the stable phase that grow independently and coalesce. These regimes are separated by a dynamic spinodal that vanishes logarithmically with system size. These results strongly suggest that our nonequilibrium, non-Hamiltonian system follows a decay mechanism very similar to the one described by the classic KJMA theory of phase transformation by nucleation and growth near a first-order equilibrium phase transition, which predicts well-defined single-droplet and multidroplet regimes. In the present far-from-equilibrium system, the desorption parameter and the distance to the coexistence point play the roles of the temperature and the external field or supersaturation, respectively. Very recently, indications of KJMA behavior have also been observed in another non-Hamiltonian, nonequilibrium system: an ecological model of invasion by exotic species 30]. We find quite exciting the strong similarity between the dynamics of metastable decay in far-from-equilibrium, non-Hamiltonian systems of applied importance, and the well-known behavior in systems that can be described by a Hamiltonian. 


\section{Acknowledgments}

We are grateful to R. M. Ziff for insightful comments. This work was supported in part

by U.S. National Science Foundation Grant No. DMR-0240078, by Florida State University through the Center from Materials Research and Technology, the School of Computational Science, and the National High Magnetic Field Laboratory, and by the Deanship of Research and Development of Universidad Simón Bolívar.

[1] K. Christmann, Introduction to Surface Physical Chemistry, Steinkopff Verlag, Darmstadt, 1991; V.P.Z. Zhdanov and B. Kazemo, Surf. Sci. Rep. 20, 111 (1994).

[2] G. C. Bond, Catalysis: Principles and Applications, Clarendon Oxford, 1987.

[3] R. M. Ziff, E. Gulari and Y. Barshad, Phys. Rev. Lett. 56, 2553 (1986).

[4] M. Ehsasi, M. Matloch, O. Frank, J. H. Block, K. Christmann, F. S. Rys and W. Hirschwald, J. Chem. Phys. 91, 4949 (1989).

[5] T. Matsushima, H. Hashimoto, and I. Toyoshima, J. Catal. 58, 303 (1979).

[6] P. Fisher and U. M. Titulaer, Surf. Sci. 221, 409 (1989).

[7] H. P. Kaukonen and R. M. Nieminen, J. Chem. Phys. 91, 4380 (1989).

[8] E. V. Albano, Appl. Phys. A 54, 2159 (1992).

[9] B. J. Brosilow and R. M. Ziff, Phys. Rev. A 46, 4534 (1992).

[10] E. Machado, G. M. Buendía, P. A. Rikvold, and R. M. Ziff, Phys. Rev. E 71, 016120 (2005).

[11] T. Tomé and R. Dickman, Phys. Rev. E 47, 948 (1993).

[12] R. M. Ziff and B. J. Brosilow, Phys. Rev. A 46, 4630 (1992).

[13] A. N. Kolmogorov, Bull. Acad. Sci. USSR, Phys. Ser. 1, 335 (1937).

[14] W. A. Johnson and R. F. Mehl, Trans. Am. Inst. Mining Metall. Eng. 135, 416 (1939).

[15] M. Avrami, J. Chem. Phys. 7, 1103 (1939); 8, 212 (1940); 9, 177 (1941).

[16] C. Borgs and R. Kotecký, J. Stat. Phys. 61, 79 (1990).

[17] D. P. Landau and K. Binder, A Guide to Monte Carlo Simulations in Statistical Physics (Cambridge Univ. Press, Cambridge, 2000), Chap. 4.2.3.3.

[18] B. Nienhuis and M. Nauenberg, Phys. Rev. Lett. 35, 477 (1975).

[19] K. Binder and D. P. Landau, Phys. Rev. B 30, 1477 (1984). 
[20] M. S. Challa, D. P. Landau and K. Binder, Phys. Rev. B 34, 1841 (1986).

[21] A. D. Bruce, J. Phys. A: Math. Gen. 18, L873 (1985).

[22] M. E. Fisher and A. N. Berker, Phys. Rev. B 26, 2507 (1982).

[23] R. A. Monetti and E. V. Albano, J. Phys. A 34, 1103 (2001).

[24] P. A. Rikvold, H. Tomita, S. Miyashita, and S. W. Sides, Phys. Rev. E 49, 5080 (1994).

[25] H. L. Richards, S. W. Sides, M. A. Novotny, and P. A. Rikvold, J. Magn. Magn. Mater. 150, 37 (1995).

[26] R. A. Ramos, P. A. Rikvold, and M. A. Novotny, Phys. Rev. B 59, 9053 (1999).

[27] M. Kolesik, M. A. Novotny, and P. A. Rikvold, Int. J. Mod. Phys. C 14, 121 (2003).

[28] M. A. Novotny, P. A. Rikvold, M. Kolesik, D. M. Townsley, and R. A. Ramos, J. Non-Cryst. Solids 274, 356 (2000).

[29] S. Frank, D. E. Roberts, and P. A. Rikvold, J. Chem. Phys., in press.

[30] G. Korniss and T. Caraco, J. Theor. Biol. 233, 137 (2005). 